\title{
CS Research Square \\ Transcriptome analysis of Nelumbo nucifera in response to antimony stress
}

\section{Yanhong Liang}

Huaibei Normal University

\section{Xiujie Zhou}

Huaibei Normal University

Qingchuan Liu

Hefei University of Technology

Fei Liu (Dliufei888@163.com )

Huaibei Normal University https://orcid.org/0000-0003-4378-5007

\section{Research Article}

Keywords: Nelumbo nucifera, antimony stress, transcriptome sequencing, differentially expressed genes

Posted Date: May 14th, 2021

DOI: https://doi.org/10.21203/rs.3.rs-502863/v1

License: @ (i) This work is licensed under a Creative Commons Attribution 4.0 International License. Read Full License 


\section{Abstract}

To explore the probable mechanism of antimony stress resistance by Nelumbo nucifera and screen out relevant antimony-resistant genes, we conducted transcriptome sequencing of Nelumbo nucifera seeds treated by antimony at low, medium, and high concentrations (100,500, $1000 \mathrm{mg} / \mathrm{L}$ respectively), screened differentially expressed genes (DEGs), and analyzed gene ontology classification and KEGG enrichment.Totally 18078 DEGs between antimony stress treatments and the control were identified, and 4192, 5850 and 8036 DEGs were found respectively.The pathways underlying the degradation of phosphopentose, limonene and pinene, the biosynthesis of flavonoid and ubiquinone, and the biosynthesis of terpenoids play critical roles in this responding process. The expressions of 7 genes validated by GPCR were consistent with the RNA-Seq results, which confirms the reliability of RNA-Seq results. This study on transcriptome analysis of Nelumbo nucifera under antimony stress provides a genetic resource and a theoretical basis for research on plant restoration of antimony pollution.

\section{Key Message}

Transcriptome analysis found that the biosynthesis of limonene and pinene, flavonoids and ubiquinone, and the biosynthesis of terpenoids play a key role in the response of Nelumbo to antimony stress.

\section{Introduction}

Antimony $(\mathrm{Sb})$ with toxicity and potential carcinogenicity is listed as a priority pollutant by many countries and international organizations. The lack of appropriate planning and management in antimony mining areas and the inappropriate use of antimony in previous years have led to the soil, water and air antimony concentrations far above state specified standards, which will seriously threaten the humanity and the whole ecosystem benign circulation. China has the largest antimony resource in the world and is faced by soil hardening and crop yield reduction due to antimony pollution, which should be solved urgently (Fei, et al. 2017; He 2012; Wang, et al. 2010). Plant restoration is a relatively economical and safe environmental restoration technology and has broad application prospects. However, due to the lack of antimony restoration plant materials, the existing studies about the effects of antimony on plants focus on the effects of antimony on early growth of plants (Baek, et al. 2014), differences in antimony accumulation among various plants (Tschan, et al. 2009), measurement of different forms of antimony in plant tissues (Tisarum, et al. 2015), and evaluation of ecological risks (Wilson, et al. 2010) and toxicity (Shtangeeva, et al. 2011; Zhong, et al. 2020). Nevertheless, the mechanism of antimony resistance by plants is unclear, which limits the technical applications of plants into restoration of antimony pollution.

Antimony existing in solutions can be easily absorbed by plants and will compete with some necessary metabolites, which thereby cause toxic hazards to plants. So far, the mechanism how plants respond to antimony stress is unknown. Recently, genomics, transcriptomics and proteomics have become increasingly important in research on the growth, development, phyletic evolution and biological toxicology of plants. Transcriptomics refers to the research on the specific cells, tissues or organs under specific growth and development stage or under certain physical conditions, and has been widely applied following the development of post-genomics (Liu, et al. 2019). When biological stress occurs, organisms can adjust relevant gene expressions, and the regulation of genetic transcription will further adjust the complex regulatory 
mechanisms during development (Lata, et al. 2018; Mittler 2006). The transcriptome sequencing based on second-generation sequencing is capable of large-scale high-throughput sequencing of total RNA (including mRNA, miRNA, IncRNA, circRNA), and thus has been extensively applied into research on transcriptome-level biological/nonbiological stress of plants and animals. Analysis of transcriptome graphs can reveal various information, including gene expressions, alternative splicing, gene structures, new genes, differentially expressed genes (DEGs), and mononucleotide fragment polymorphyism, and can annotate the obtained genes and further interpret the molecular mechanisms of plant actions under unfavourable conditions.

Bowl lotus is a perennial aquatic plant with multisectional rhizome, and has long and thick underground stems. Bowl lotus growing throughout China can be extensively planted in lakes and rivers and be used into water landscaping, scenery appreciation, and water purification. Bowl lotus seeds can germinate as long as the seed shells can absorb enough water and swell. The short period of germination is favorable for experimental observation. In this study targeting at Nelumbo nucifera seeds, the DEGs of Nelumbo nucifera under stress of different antimony concentrations were investigated by transcriptomics sequencing and real-time quantitative polymerase chain reaction (RT-qPCR). Then the relevant genes of bowl locus under antimony stress were screened out, and key regulatory genes were recognized.

\section{Materials And Methods}

\subsection{Materials}

High-quality Nelumbo nucifera seeds with basically the same saturation degree were selected. After opening, the seeds were cleaned with distilled water 3-4 times, soaked in $2 \%$ hypochlorous acid for 10 min, washed with distilled water 3-4 times and soaked in clear water for 24 hours. The soaked seeds were placed into glass vessels (8 seeds/vessel) and treated with different concentrations of antimony (seeds soaked with clean water serving as the control): low, medium and high concentrations: $100 \mathrm{mg} / \mathrm{L}$ (T100), $500 \mathrm{mg} / \mathrm{L}$ (T100) and 1000 $\mathrm{mg} / \mathrm{L}(\mathrm{T} 1000)$ respectively. During the 7 days of treatments at $25^{\circ} \mathrm{C}$, seed germination of each group was observed every day. Each treatment was biologically repeated 3 times. After that, the samples with basically the same growing conditions were selected and stored in liquid nitrogen and dry ice until used.

\subsection{Total RNA extraction and transcriptome sequencing}

After fast freezing in liquid nitrogen, the samples stored in dry ice were sent to Shanghai Meiji Biology Co., Ltd. for total RNA extraction and sequencing. Total RNA was extracted from each sample and then its concentration and purity were detected using a Nanosrop2000 device. RNA integrity was tested by agarose gel electrophoretic analysis, and RIN was monitored by an Agilent2100 instrument. Magnetic beads containing Oligo(dT) were used for A-T base matching with polyA, and mRNA was thereby isolated from the total RNA. Then a fragmentation buffer was added, and the mRNA was randomly broken into small fragments in size of $300 \mathrm{bp}$. Under the action of reverse transcriptase, random hexamers were added, and the single strand of cDNA was synthesized via reverse-transcription with mRNA as the template. After that, the second strand was synthesized, forming a stable double-strand structure. An End Repair Mix was added to complement the double-strand cDNA into a flat end. Then the 3 ' end was added with -"A" for connecting the Y-shaped head (sequence of head: 5 ': AGATCGGAAGAGCACACGTC 3': AGATCGGAAGAGCGTCGTGT). 
The sequence signals were converted, via CASAVA base calling on Illumina, into text signals, which were stored as the raw data in the format of fastq. The raw data contained the sequences of the sequencing heads, lowquality fragments, high-N (N: uncertain information) sequences, and over-short sequences, which will severely lower the quality of subsequent analysis. Hence, the original sequencing data were sent into quality control in advance, so that high-quality clean data were obtained, which ensured the accuracy of the subsequent analysis.

Sequencing was conducted by using an Illumina TruseqTM RNA sample kit, with quantification by TBS380. The sequences were subjected to the machines in the mixing form. The cBot was sent to bridge-typed PCR amplification, forming clusters. Sequencing was performed on Illumina (PE library, reading length 2*150bp). Cutting was conducted on SeqPrep and Sickle. The heads, repeated or redundant sequences, blur bases, and reads in length $<60 \mathrm{bp}$ (reads except 15-34nt on miRNAs) were removed from the raw data. The sequences were matched with the genome of Nelumbo nucifera on TopHat2.0. After quality control and cutting, the data were subjected to statistical analysis and quality assessment again, including statistics of distributions of (1) A/T/G/C concentrations, (2) base quality, and (3) base error rates. Based on the known reference genome, the mapped reads were assembled and spliced on Cufflinks, and compared with some known transcripts. In this way, the transcripts without annotations were obtained, and the functions of the potential new transcripts were annotated.

\subsection{Screening of DEGs in Nelumbo nucifera under antimony stress}

Firstly, the gene expressions in the samples were assessed using TPM. Specifically, first the gene lengths and then the sequencing depths were uniformized, so that the total expression levels among different samples were consistent, which helped with the more visual comparison of expression levels among genes. The expression levels of genes and transcripts were quantitatively analyzed on RSEM, and DEGs among different processed samples were identified. Moreover, the DEGs were tested by significance analysis $(P<0.05)$. With a blank without antimony stress treatment as the control, the up- or down-regulation of genes in the low-, medium- and high-antimony groups was statistically analyzed.

\subsection{Functions and annotations of DEGs}

The DEGs as-obtained were subjected to gene ontology (GO) enrichment analysis on GOseq at the significant level of $P \leq 0.05$. From the $\mathrm{GO}$ analysis, the annotations of the DEGs in the KEGG database can be determined, so as to understand the major metabolic pathways and signal transduction pathways involving these DEGs.

\subsection{RT-qPCR of DEGs}

The reliability of the transcriptome data was validated via RT-qPCR. With the 4-fold dilute of the reversetranscribed cDNA as the template, the DEGs with $\mid \log 2$ (fold-change) $\mid \geq 1$ or $\leq 0.5$ in the transcriptome sequences were selected and sent to RT-qPCR. According to the sequences as-obtained, primers were designed on Primer premier 6.0 (Biosoft International, Palo Alto, CA, USA), and the samples were validated by RT-qPCR. Each gene amplification was accompanied with the simultaneous amplification of the internal reference gene ( $\beta$-Actin). The sequences of the genes and their primers were listed in Table 1. 
Table 1

Different gene primers of Nelumbo nucifera in response to antimony stress

\begin{tabular}{|llll|}
\hline gene name & Forward primer & Reverse primer & Product length \\
\hline$\beta$-actin & CTGAACCCAAAGGCTAATCG & ACTGGCGTAGAGGGAAAGAA & 108 \\
\hline LOC104601801 & TGGGCAGGCTTACTCGTTTT & TTAGCAACAAGATGGCCGCT & 129 \\
\hline LOC104585725 & CGTTCAAGTCAGATGCGCTG & TTCGGACATAAAACCGGCCA & 205 \\
\hline LOC104607195 & ACTCCCGATGTCAATGCCTC & TTGGGGTCACAACACCAGAG & 101 \\
\hline LOC104610071 & TTCAAGTCAGATGCGCTGGT & CCAACCTGGTTCTTCGGACA & 215 \\
\hline LOC104603985 & ATGGGCAGGATGCGGTTTAT & GGTTCCTGGTCTGCCATCAA & 176 \\
\hline LOC104588398 & AAGCTCAGTGGTTCAGTCGG & CCCACACTCCAGTCATCACC & 177 \\
\hline
\end{tabular}

\section{Results And Analysis}

\subsection{Sequencing data and quality analysis}

The transcriptomes from different groups were subjected to high-throughput sequencing on IlluminaHiseq, and the characteristics of the DEGs as-obtained were analyzed. Totally $73046 \mathrm{~Gb}$ clean data were obtained, and the amount of clean data from each sample was up to $6.28 \mathrm{bG}$. The error rate below $0.025 \mathrm{Q} 20$ was larger than $98 \%$, and that below Q30 was above 94\%. These results indicate our transcriptome data are of high quality and meet the basic requirements for transcriptome analysis (Table 2 ).

Table 2

Evaluation of sequencing data quality of Nelumbo nucifera

\begin{tabular}{|lllllllll|}
\hline Sample & $\begin{array}{l}\text { Raw } \\
\text { reads }\end{array}$ & Raw bases & $\begin{array}{l}\text { Clean } \\
\text { reads }\end{array}$ & Clean bases & $\begin{array}{l}\text { Error } \\
\text { rate }\end{array}$ & Q20 & Q30 & $\begin{array}{l}\text { GC } \\
\text { content }\end{array}$ \\
\hline CK & 51500230 & 7776534730 & 50979848 & 7630742760 & 0.0244 & 98.27 & 94.75 & 45.53 \\
\hline T100_1 & 48755934 & 7362146034 & 48127280 & 7187919410 & 0.0244 & 98.25 & 94.75 & 45.8 \\
\hline T100_2 & 53089540 & 8016520540 & 52402138 & 7832822614 & 0.0244 & 98.28 & 94.8 & 46.36 \\
\hline T100_3 & 49019214 & 7401901314 & 48253482 & 7134490812 & 0.0242 & 98.32 & 94.91 & 46.98 \\
\hline T500_1 & 47908962 & 7234253262 & 47321574 & 7086056882 & 0.0249 & 98.08 & 94.26 & 45.84 \\
\hline T500_2 & 51768128 & 7816987328 & 51160910 & 7660298314 & 0.0244 & 98.26 & 94.71 & 45.59 \\
\hline T500_3 & 46189372 & 6974595172 & 45580696 & 6815525189 & 0.0246 & 98.2 & 94.57 & 45.53 \\
\hline T1000_1 & 50346474 & 7602317574 & 49760484 & 7452432367 & 0.0245 & 98.24 & 94.67 & 45.7 \\
\hline T1000_2 & 47664754 & 7197377854 & 47187202 & 7074883571 & 0.0244 & 98.27 & 94.69 & 45.9 \\
\hline T1000_3 & 51196662 & 7730695962 & 50626748 & 7580384175 & 0.0245 & 98.23 & 94.66 & 45.63 \\
\hline
\end{tabular}




\subsection{Nelumbo nucifera sequence alignment and annottions}

The clean reads were matched with the reference genes on Hisat2, and the database of the reference genes was Nelumbo_nucifera. The maatching rate was above $92.96 \%$. The proportion of multiple sequences was $1.39-$ $1.54 \%$, and the proportion of single sequences was above $91 \%$ (Table 3 ).

Table 3

Statistics of sequence alignment results of Nelumbo nucifera

\begin{tabular}{|lllll|}
\hline Sample & Total reads & Total mapped & Multiple mapped & Uniquely mapped \\
\hline CK & 50979848 & $48948743(96.02 \%)$ & $756279(1.48 \%)$ & $48192464(94.53 \%)$ \\
\hline T100_1 & 48127280 & $45702082(94.96 \%)$ & $669554(1.39 \%)$ & $45032528(93.57 \%)$ \\
\hline T100_2 & 52402138 & $48712887(92.96 \%)$ & $805522(1.54 \%)$ & $47907365(91.42 \%)$ \\
\hline T100_3 & 48253482 & $45085709(93.44 \%)$ & $696559(1.44 \%)$ & $44389150(91.99 \%)$ \\
\hline T500_1 & 49760484 & $47008163(94.47 \%)$ & $722034(1.45 \%)$ & $46286129(93.02 \%)$ \\
\hline T500_2 & 47187202 & $44204078(93.68 \%)$ & $658412(1.4 \%)$ & $43545666(92.28 \%)$ \\
\hline T500_3 & 50626748 & $47568553(93.96 \%)$ & $741858(1.47 \%)$ & $46826695(92.49 \%)$ \\
\hline T1000_1 & 47321574 & $44138495(93.27 \%)$ & $682117(1.44 \%)$ & $43456378(91.83 \%)$ \\
\hline T1000_2 & 51160910 & $48551832(94.9 \%)$ & $728792(1.42 \%)$ & $47823040(93.48 \%)$ \\
\hline T1000_3 & 45580696 & $43032382(94.41 \%)$ & $652022(1.43 \%)$ & $42380360(92.98 \%)$ \\
\hline
\end{tabular}

The genes and transcripts as-obtained were compared with some major databases (NR, Swiss-Prot, Pfam, EggNOG, GO, KEGG), so as to fully identify the functions of the genes and transcripts and to statistically analyze the annotations based on these databases (Table 3). Totally 30423 expressed genes were detected, including 5261 new genes and 25162 known genes, accounting for $82.7 \%$ of all genes. Totally 64241 expressed transcripts were identified, including 23368 new transcripts and 40873 known transcripts, accounting for $63.62 \%$ of all transcripts (Table 4). These data suggest the functions of many genes of Nelumbo nucifera have not been fully defined. 
Table 4

Transcriptome functional annotation results

\begin{tabular}{|lllll|}
\hline & $\begin{array}{l}\text { Expre_Gene } \\
\text { number(percent) }\end{array}$ & $\begin{array}{l}\text { Expre_Transcript } \\
\text { number } \\
\text { (percent) }\end{array}$ & $\begin{array}{l}\text { All_Gene } \\
\text { number } \\
\text { (percent) }\end{array}$ & $\begin{array}{l}\text { All_Transcript } \\
\text { number } \\
\text { (percent) }\end{array}$ \\
\hline GO & $11932(0.4742)$ & $17971(0.4397)$ & $12373(0.437)$ & $18945(0.4153)$ \\
\hline KEGG & $9808(0.3898)$ & $15479(0.3787)$ & $10303(0.3639)$ & $16506(0.3619)$ \\
\hline COG & $22137(0.8798)$ & $34902(0.8539)$ & $23631(0.8347)$ & $37637(0.8251)$ \\
\hline NR & $22458(0.8925)$ & $35300(0.8636)$ & $24026(0.8486)$ & $38139(0.8361)$ \\
\hline Swiss-Prot & $18781(0.7464)$ & $29571(0.7234)$ & $19930(0.704)$ & $31769(0.6965)$ \\
\hline Pfam & $19894(0.7906)$ & $31276(0.7652)$ & $21164(0.7476)$ & $33652(0.7378)$ \\
\hline Total_anno & $22465(0.8928)$ & $35307(0.8638)$ & $24033(0.8489)$ & $38147(0.8363)$ \\
\hline Total & $25162(1.0)$ & $40875(1.0)$ & $28311(1.0)$ & $45614(1.0)$ \\
\hline
\end{tabular}

\subsection{Function annotations of DEGs}

Together with the GO database, the DEGs from the 3 antimony-treated groups were classified. Macroscopically, three categories were identified: bioprocesses, cell components, and molecular functions. Then the top $20 \mathrm{GO}$ terms ranked by the significance (the smallest p-values) of GO enrichment were displayed in Fig. 1. Since the same transcriptome may be mapped to different nodes, among all 21065 terms of bioprocesses, accounting for $40.6 \%$, the largest numbers of unigene were involved in metabolic processes $(4819,22.88 \%)$ and cell processes $(5187,24.62 \%)$. There were 20061 terms of cell components, accounting for $38.67 \%$, and the largest numbers of unigene were involved in cells $(4243,21.15 \%)$ and cellular components (4202, 20.95\%). There were 10752 terms of molecular functions, accounting for $20.73 \%$, and the largest numbers of unigene were involved in catalytic activity (4179, 38.87\%) and binding proteins (4884, 45.42\%) (Fig. 1).

\subsection{Screening and annotation of DEGs in Nelumbo nucifera under antimony stress}

The threshold of DEG selection was $|\log 2 \mathrm{FC}| \geq 1$ or $\mathrm{P}<0.05$, and the analytical software was edgeR. In T100, totally 4902 DEGs were found, including 1556 upregulated genes and 3346 downregulated genes. In T500, totally 5769 DEGs were identified, including 2324 upregulated genes and 3445 downregulated genes. In T1000, totally 8253 DEGs were recognized, including 3103 upregulated genes and 5150 downregulated genes (Fig. 2).

Moreover, the 3 groups (T100, T500, T1000) shared 21 DEGs (including 4 upregulated genes and 17 downregulated genes), and their differential expressions were above 1000 times (|log2FC| $\geq 10$ ). These DEGs were plotted on Venn diagrams (Fig. 3). As for the upregulated DEGs, T100, T500 and T1000 had 12, 11 and 10 exclusive genes respectively (Fig. 3A). As for the downregulated DEGs, T100, T500 and T1000 had 9, 10 and 67 exclusive genes respectively (Fig. 3B).

\subsection{KEGG annotations and metabolic pathways of DEGs in Nelumbo nucifera under antimony stress}


The DEGs of Nelumbo nucifera were matched with the KEGG database, and were annotated into six first-level categories and 25 second-level categories, which involved 142 metabolic pathways. The pathways that each involved more than 10 DEGs were mainly from six aspects, including genetic information processing, metabolic processes, cellular processes, environmental information processing, biosystems, and human diseases (Fig. 4). Among these six aspects, the dominant one was metabolism, including carbohydrate metabolism, amino acid metabolism, amino acid folding/classification/degradation, lipid metabolism, nucleotide metabolism, and metabolism of other secondary substances. Only two metabolic pathways involved human diseases: endocrine metabolic diseases, and drug tolerance: antimicrobial. KEGG analysis of antimony stress showed the involvement of gene changes in human diseases, indicating antimony affected the endocrine metabolism and drug tolerance of humans to some extent.

\section{Note}

Y-axis: name of KEGG metabolic pathway; X-axis: number of genes/transcripts annotated to this pathway. The seven categories of KEGG metabolic pathways include metabolism, genetic information processing, environmental information processing, cellular processes, organismal systems, human diseases, and drug development. (note: the number of categories differs among different species).

The pathway enrichment analysis showed the above 21 DEGs responding to antimony stress mainly involved carbohydrate metabolism (5.27\%), terpenoid and polyketide compound metabolism (0.09\%), amino acid metabolism $(0.53 \%)$, biosynthesis of other secondary metabolites $(1.28 \%)$, and metabolism of auxiliary factors and vitamins (0.45\%). The concrete metabolic pathways include Pentose phosphate pathway (6PGL, EC:3.1.1.31), Limonene and pinene degradation, Flavonoid biosynthesis, Tryptophan metabolism, Ubiquinone and other terpenoid- quinone biosynthesis (VTE4, EC:2.1.1.95), Isoquinoline alkaloid biosynthesis, Pentose and glucuronate interconversions (PME53, EC:3.1.1.11), and Starch and sucrose metabolism.

\subsection{RT-qPCR of DEGs in Nelumbo nucifera}

To validate the accuracy of the transcription results, we selected 6 DEGs with significant changes, and designed

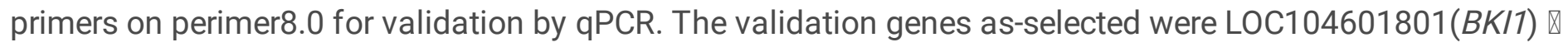
LOC104585725(GH3.5) LOC104607195(PIF3) 『LOC104610071(ETR2) \LOC104603985(PYL8) 》 LOC104588398(GID1C). The qTR-PCR results of these 6 genes are highly consistent with their expression trends detected by transcription analysis.

\section{Discussion}

The intake of heavy metals, which are non-necessary elements for plant growth, will interfere with the normal growth of plants and threaten the health of humans and ecosystems through food chains. Heavy metal stress will affect a series of reactions in plants, including gene expressions under stress conditions, cell metabolisms, growth and development. Transcriptomics analysis deepens our knowledge on the responses of plants to heavy metal stress and helps to discuss the possibility of heavy metal detoxifcation by plants. Previous studies about the effects of $\mathrm{Cd}$ on rice indicate $\mathrm{Cd}$ can induce metabolism and ATP-related regulatory proteins in roots, and significant upregulation of proteins related with antioxidant Cd stress in seeds (Ahsan, et al. 2007; Chen, et al. 2018). For castors under $\mathrm{Cd}$ stress, the genes related with proline and soluble phenol are upregulated, and thus, 
these substances become are permeable and will be more absorbed by plants, which improve the heavy metal tolerance of plants to some extent (Liu, et al. 2011). When plants respond to drought, high salts, heavy metals and other types of environmental stress, the growth-related genes will be downregulated to enhance the stress resistance (Gong, et al. 2020). Antimony can inhibit the early growth of Chinese cabbages, rapes, wheat and cucumbers (Baek, et al. 2014). The upregulation of BRs will promote the synthesis of brassinosteroids and thereby relieve the toxicity of antimony stress to Arabidopsis thaliana (Wu, et al. 2019). The above studies indicate that plants will use different adaptation mechanisms in response to heavy metal stress. In the present study, the number of DEGs increases with the rise of antimony concentration, and is larger under high concentration treatment (T1000) than under low concentration treatment (T100), and the number of downregulated genes is significantly larger than that of upregulated genes $(P \leq 0.05)$ (Fig. 2). Hence, it is deduced that Nelumbo nucifera is not sensitive to antimony mass concentrations, and the majority of DEGs will be differentially expressed only under specific mass concentrations. Bowl lotus responds to antimony stress mainly by downregulating DEGs. When the differential expression was magnified by 1000 times, 21 genes were screened out (Table 5), indicating these genes are critical in the response to antimony stress by Nelumbo nucifera and can serve as key candidate genes in the response of Nelumbo nucifera to antimony stress. These functions will be validated in future research. 
Table 5

Important candidate genes in response to antimony stress in Nelumbo nucifera

\begin{tabular}{|c|c|c|}
\hline Express trend & Gene name & Gene Description \\
\hline down & LOC104590835 & probable tocopherol O-methyltransferase, chloroplastic \\
\hline down & LOC104595104 & probable (S)-N-methylcoclaurine 3'-hydroxylase isozyme 2 \\
\hline down & LOC104608209 & non-classical arabinogalactan protein 31-like \\
\hline down & LOC104593458 & probable membrane-associated kinase regulator 2 \\
\hline down & LOC104598560 & 14 kDa proline-rich protein DC2.15-like \\
\hline down & LOC104597689 & subtilisin-like protease SBT3.18 \\
\hline down & LOC104597387 & myosin-9, transcript variant $\mathrm{X} 1$ \\
\hline down & LOC104587630 & FT-interacting protein 1 \\
\hline down & LOC104598507 & aldo-keto reductase family 4 member C9-like \\
\hline down & LOC104610882 & cytochrome P450 77A3 \\
\hline down & LOC104587648 & GDSL esterase/lipase At5g33370-like \\
\hline down & LOC104596714 & flavonol synthase/flavanone 3-hydroxylase-like \\
\hline down & LOC104611679 & G2/mitotic-specific cyclin C13-1-like \\
\hline down & LOC104605808 & probable 6-phosphogluconolactonase 1 , transcript variant $\mathrm{X} 1$ \\
\hline down & LOC104604473 & probable pectinesterase 53 \\
\hline down & LOC104597387 & myosin-9, transcript variant $\mathrm{X} 1$ \\
\hline down & LOC104592804 & bidirectional sugar transporter SWEET12-like \\
\hline up & LOC104604327 & uncharacterized LOC104604327 \\
\hline up & LOC104604928 & thioredoxin-like protein CXXS1 \\
\hline up & LOC104586052 & uncharacterized LOC104586052 \\
\hline up & LOC104596739 & uncharacterized LOC104596739 \\
\hline
\end{tabular}

KEGG metabolic pathway analysis shows that the DEGs are enriched in flavonoid biosynthesis, pentose and gluconic salt interconversion, isoquinoline biosynthesis, phosphopentose pathway, and biosynthesis of ubiquinone and other terpenoid-quinones (Fig. 4). The top 20 terms of significant DGE enrichment include pectinesterase (PME53), tocopherol-methyl transferase (VTE4), 6-phosphogluconate (6PGL), and flavonoid3hydroxylase $(F 3 H)$. Secondary metabolites are a group of small-molecular organic compounds that are produced by secondary metabolism and not needed by the growth and development of plants. In response to environmental stress, the secondary metabolism of plants will be further induced, and more metabolites will be produced to improve the surviving and self-protecting abilities. This is a result from the interaction of plants with biological and nonbiological factors during long-term evolution (Choi, et al. 2014; Guerriero, et al. 2018; 
Mishra, et al. 2019). The secondary metabolites of plants can be divided into phenylpropanoids, terpenoids, flavonoids, quinones, tannins, and sterides (Bouwmeester, et al. 2003). The environmental stress of nutrition deficiency will promote the production of secondary metabolites in plants (Bais 2010; Grove, et al. 2012). The concentrations of phenols produced by marigolds under drought stress are significantly larger than those under water sufficiency (Tang, et al. 1994). Under high-concentration Pb treatment, the concentrations of key enzymes in secondary metabolites and secondary metabolism activities of dry grasses increase (Wang, et al. 2011). The concentrations of trans-anethole and oxygen-containing compounds significantly decrease, and the concentrations of limonene and monoterpenes considerably rise (Lima, et al. 2013). Bowl lotus in response to antimony stress will downregulate the genes related with secondary metabolism, so as to lower the biosynthesis of limonene, pinene and quinones.

Flavonoids are an important group of antibacterial and anti-stress compounds in plants, and are excellent antioxidants that can remove $\mathrm{H}_{2} \mathrm{O}_{2}$ around cell membranes. The water extracts of flavonoids from Ipomoea aquatica Forsk and Enydra fluctuans Lour. can clear away free radicals and improve the activities of glutathione-S- transferase, glutathione reductase, catalase and superoxide dismutase, thereby enhancing the antioxidant capacity in vivo (Dua, et al. 2015). F3H is a key enzyme in the synthesis of flavonoids, and the gene $\mathrm{F} 3 \mathrm{H}$ controlling the synthesis of this enzyme is critical in the flower color formation of plants and in the stress resistance of plants (Mahajan and Yadav 2014; Song, et al. 2016). During the response of Nelumbo nucifera to antimony stress, $\mathrm{F} 3 \mathrm{H}$ is downregulated in the biosynthesis of flavonoids, and the expression levels are more than 1000 times different between T1000 and the control ( $|\log 2 \mathrm{FC}| \geq 10$ ) (Fig. 5), and other relevant genes including $F L S 1$ and $F L$ are downregulated, indicating flavonoids play an important role in the response of Nelumbo nucifera to antimony stress.

The phosphopentose pathway, a key pathway of sugar metabolism in plants, is closely related to various nonbiological stress, growth and development of plants. This pathway is involved in the early response of plants to non-biological stress (Cardi, et al. 2011; Pandey, et al. 2015). As a metabolic sensor under oxygen stress, it is pivotal in balancing oxido-reduction in cells (Gruning, et al. 2011; Kruger, et al. 2011; Rokitta, et al. 2012). 6PGL is an enzyme in catalysis of the phosphopentose pathway, and can catalyze the hydrolysis of 6PGL. The activity of 6PGL is not necessary for the normal growth and propagation of yeast cells (Murthi, et al. 2010; Stanford, et al. 2004). The size of 6PGL mutant strains in Arabidopsis thaliana is about half that in wide-type strains, indicating 6PGL can affect the size of plants (Xiong, et al. 2009). When temperature sharply drops, the relative expression level of 6PGL is suddenly increased, which enhances the phosphopentose pathway. On the one hand, this pathway increases the production of some intermediates (e.g. RuBP, NADPH), providing raw materials and exclusive donors to the growth of plants and ensuring the production and utilization of ATP. On the other hand, accumulation of 6-phosphogluconate, the final product of the phosphopentose pathway, will intensify cell osmotic pressure and enhance freezing resistane of plants (Savitch, et al. 2000; Stincone, et al. 2015). Wheat will relieve the harms of Al stress by increasing the production of enzymes related to the phosphopentose pathway (Zhang, et al. 2013). It is deduced that in the response of Nelumbo nucifera to antimony stress, the genes related with the phosphopentose pathway are downregulated, so as to relieve the harms of antimony stress to Nelumbo nucifera.

\section{Conclusions}


The transcriptomes of Nelumbo nucifera under different levels of antimony stress were analyzed by lluminaHISEq. As the antimony concentration rose, the number of DEGs in Nelumbo nucifera increased. These DEGs are mainly enriched in carbohydrate metabolism, amino acid metabolism, amino acid folding/classification/degradation, lipid metabolism, nucleotide metabolism, and metabolism of other secondary substances. In the response to antimony stress, the degradation of phosphopentose, limonene, pinene, and the biosynthesis of flavonoids, ubiquinone and other terpenes are very important. From the DEGs, 21 candidate genes that may be closely related to the response of Nelumbo nucifera to antimony stress were screened out, which will offer a gene resource and a theoretical basis for further functional validation and deep research on these DEGs.

\section{Declarations}

\section{Compliance with ethical standards}

Conflict of interest The authors declare that the research was conducted in the absence of any commercial or financial relationships that could be construed as a potential conflict of interest.

Contributor Roles Taxonomy Conceived and designed the experiments: XJZ FL. Performed the experiments: YHL QCL XJZ. Analyzed the data: YHL QCL XJZ FL. Contributed reagents/materials/analysis tools: YHL QCL XJZ. Wrote the paper: YHL. Plotting: YHL QCL. All authors read and approved the final manuscript.

\section{References}

Ahsan N, Lee SH, Lee DG, Lee H, Lee SW, Bahk JD, Lee BH (2007) Physiological and protein profiles alternation of germinating rice seedlings exposed to acute cadmium toxicity. C R Biol 330:735-746

Baek YW, Lee WM, Jeong SW, An YJ (2014) Ecological effects of soil antimony on the crop plant growth and earthworm activity. Environ Earth Sci 71:895-900

Bais HP (2010) Allelopathy and exotic plant invasion: From molecules and genes to species interactions (September, pg 1377, 2003). Science 327:781-781

Bouwmeester HJ, Matusova R, Zhongkui S, Beale MH (2003) Secondary metabolite signalling in host-parasitic plant interactions. Current Opinion in Plant Biology 6:358-364

Cardi M, Chibani K, Cafasso D, Rouhier N, Jacquot JP, Esposito S (2011) Abscisic acid effects on activity and expression of barley (Hordeum vulgare) plastidial glucose-6-phosphate dehydrogenase. J Exp Bot 62:40134023

Chen Q, Lu X, Guo X, Pan Y, Yu B, Tang Z, Guo Q (2018) Differential responses to Cd stress induced by exogenous application of $\mathrm{Cu}, \mathrm{Zn}$ or $\mathrm{Ca}$ in the medicinal plant Catharanthus roseus. Ecotoxicol Environ Saf 157:266-275

Choi SH, Park S, Lim YP, Kim SJ, Park JT, An G (2014) Metabolite profiles of glucosinolates in cabbage varieties (Brassica oleracea var. capitata) by season, color, and tissue position. Hortic Environ Biote 55:237-247 
Dua TK, Dewanjee S, Khanra R, Bhattacharya N, Bhaskar B, Zia-Ul-Haq M, De Feo V (2015) The effects of two common edible herbs, Ipomoea aquatica and Enhydra fluctuans, on cadmium-induced pathophysiology: a focus on oxidative defence and anti-apoptotic mechanism. J Transl Med 13:

Fei JC, Min XB, Wang ZX, Pang Zh, Liang YJ, Ke Y (2017) Health and ecological risk assessment of heavy metals pollution in an antimony mining region: a case study from South China. Environ Sci Pollut R 24:2757327586

Gong Z, Xiong L, Shi H, Yang S, Herrera-Estrella LR, Xu G, Chao DY, Li J, Wang PY, Qin F, Li J, Ding Y, Shi Y, Wang Y, Yang Y, Guo Y, Zhu JK (2020) Plant abiotic stress response and nutrient use efficiency. Sci China Life Sci 63:635-674

Grove S, Haubensak KA, Parker IM (2012) Direct and indirect effects of allelopathy in the soil legacy of an exotic plant invasion. Plant Ecol 213:1869-1882

Gruning NM, Rinnerthaler M, Bluemlein K, Mulleder M, Wamelink MMC, Lehrach H, Jakobs C, Breitenbach M, Ralser M (2011) Pyruvate Kinase Triggers a Metabolic Feedback Loop that Controls Redox Metabolism in Respiring Cells. Cell Metab 14:415-427

Guerriero G, Berni R, Munoz-Sanchez JA, Apone F, Abdel-Salam EM, Qahtan AA, Alatar AA, Cantini C, Cai G, Hausman JF, Siddiqui KS, Hernandez-Sotomayor SMT, Faisal M (2018) Production of Plant Secondary Metabolites: Examples, Tips and Suggestions for Biotechnologists. Genes-Basel 9:

He MCW, X. Q.Wu, F. C.Fu, Z. Y. (2012) Antimony pollution in China. Science of the Total Environment 421:41-50

Kruger A, Gruning NM, Wamelink MMC, Kerick M, Kirpy A, Parkhomchuk D, Bluemlein K, Schweiger MR, Soldatov A, Lehrach H, Jakobs C, Ralser M (2011) The Pentose Phosphate Pathway Is a Metabolic Redox Sensor and Regulates Transcription During the Antioxidant Response. Antioxid Redox Sign 15:311-324

Lata R, Chowdhury S, Gond SK, White JF, Jr. (2018) Induction of abiotic stress tolerance in plants by endophytic microbes. Lett Appl Microbiol 66:268-276

Lima NGPB, De Sousa DP, Pimenta FCF, Alves MF, De Souza FS, Macedo RO, Cardoso RB, de Morais LCSL, Diniz MDFM, de Almeida RN (2013) Anxiolytic-like activity and GC-MS analysis of (R)-(+)-limonene fragrance, a natural compound found in foods and plants. Pharmacol Biochem Be 103:450-454

Liu CF, Guo JL, Cui YL, Lu TF, Zhang XH, Shi GR (2011) Effects of cadmium and salicylic acid on growth, spectral reflectance and photosynthesis of castor bean seedlings. Plant Soil 344:131-141

Liu L, Li QZ, Jin W, Lv H, Lin H (2019) Revealing Gene Function and Transcription Relationship by Reconstructing Gene-Level Chromatin Interaction. Comput Struct Biotec 17:195-205

Mahajan M, Yadav SK (2014) Overexpression of a tea flavanone 3-hydroxylase gene confers tolerance to salt stress and Alternaria solani in transgenic tobacco. Plant Mol Biol 85:551-573

Mishra B, Chand S, Singh Sangwan N (2019) ROS management is mediated by ascorbate-glutathione-atocopherol triad in co-ordination with secondary metabolic pathway under cadmium stress in Withania 
Mittler R (2006) Abiotic stress, the field environment and stress combination. Trends Plant Sci 11:15-19

Murthi A, Shaheen HH, Huang HY, Preston MA, Lai TP, Phizicky EM, Hopper AK (2010) Regulation of tRNA Bidirectional Nuclear-Cytoplasmic Trafficking in Saccharomyces cerevisiae. Mol Biol Cell 21:639-649

Pandey P, Ramegowda V, Senthil-Kumar M (2015) Shared and unique responses of plants to multiple individual stresses and stress combinations: physiological and molecular mechanisms. Frontiers in Plant Science 6:

Rokitta SD, John U, Rost B (2012) Ocean Acidification Affects Redox-Balance and lon-Homeostasis in the LifeCycle Stages of Emiliania huxleyi. Plos One 7:

Savitch L, Harney T, Huner N (2000) Sucrose metabolism in spring and winter wheat in response to high irradiance, cold stress and cold acclimation. Physiologia Plantarum 108:270-278

Shtangeeva I, Bali R, Harris A (2011) Bioavailability and toxicity of antimony. J Geochem Explor 110:40-45

Song XY, Diao JJ, Ji J, Wang G, Guan CF, Jin C, Wang YR (2016) Molecular cloning and identification of a flavanone 3-hydroxylase gene from Lycium chinense, and its overexpression enhances drought stress in tobacco. Plant Physiol Bioch 98:89-100

Stanford DR, Whitney ML, Hurto RL, Eisaman DM, Shen WC, Hopper AK (2004) Division of Labor Among the Yeast Sol Proteins Implicated in tRNA Nuclear Export and Carbohydrate Metabolism. Genetics 168:117-127

Stincone A, Prigione A, Cramer T, Wamelink MMC, Campbell K, Cheung E, Olin-Sandoval V, Gruning NM, Kruger A, Alam MT, Keller MA, Breitenbach M, Brindle KM, Rabinowitz JD, Ralser M (2015) The return of metabolism: biochemistry and physiology of the pentose phosphate pathway. Biol Rev 90:927-963

Tang C-S, Cai W-F, Kohl K, Nishimoto RK (1994) Plant Stress and Allelopathy. In: Allelopathy. American Chemical Society, pp 142-157

Tisarum R, Ren JH, Dong XL, Chen H, LessI JT, Ma LNQ (2015) A new method for antimony speciation in plant biomass and nutrient media using anion exchange cartridge. Talanta 144:1171-1175

Tschan M, Robinson BH, Nodari M, Schulin R (2009) Antimony uptake by different plant species from nutrient solution, agar and soil. Environmental Chemistry 6:

Wang C, Lu J, Zhang SH, Wang PF, Hou J, Qian J (2011) Effects of Pb stress on nutrient uptake and secondary metabolism in submerged macrophyte Vallisneria natans. Ecotox Environ Safe 74:1297-1303

Wang XQ, He MC, Xie J, Xi JH, Lu XF (2010) Heavy metal pollution of the world largest antimony mine-affected agricultural soils in Hunan province (China). J Soil Sediment 10:827-837

Wilson SC, Lockwood PV, Ashley PM, Tighe M (2010) The chemistry and behaviour of antimony in the soil environment with comparisons to arsenic: A critical review. Environ Pollut 158:1169-1181 
Wu CC, Li F, Xu H, Zeng WM, Yu RL, Wu XL, Shen L, Liu YD, Li JK (2019) The potential role of brassinosteroids (BRs) in alleviating antimony (Sb) stress in Arabidopsis thaliana. Plant Physiol Bioch 141:51-59

Xiong Y, DeFraia C, Williams D, Zhang X, Mou Z (2009) Characterization of Arabidopsis 6Phosphogluconolactonase T-DNA Insertion Mutants Reveals an Essential Role for the Oxidative Section of the Plastidic Pentose Phosphate Pathway in Plant Growth and Development. Plant and Cell Physiology 50:12771291

Zhang L, Liu J, Wang XM, Bi YR (2013) Glucose-6-phosphate dehydrogenase acts as a regulator of cell redox balance in rice suspension cells under salt stress. Plant Growth Regul 69:139-148

Zhong QY, Ma CL, Chu JW, Wang XL, Liu XT, Ouyang W, Lin CY, He MC (2020) Toxicity and bioavailability of antimony in edible amaranth (Amaranthus tricolor Linn.) cultivated in two agricultural soil types. Environ Pollut 257:

\section{Figures}
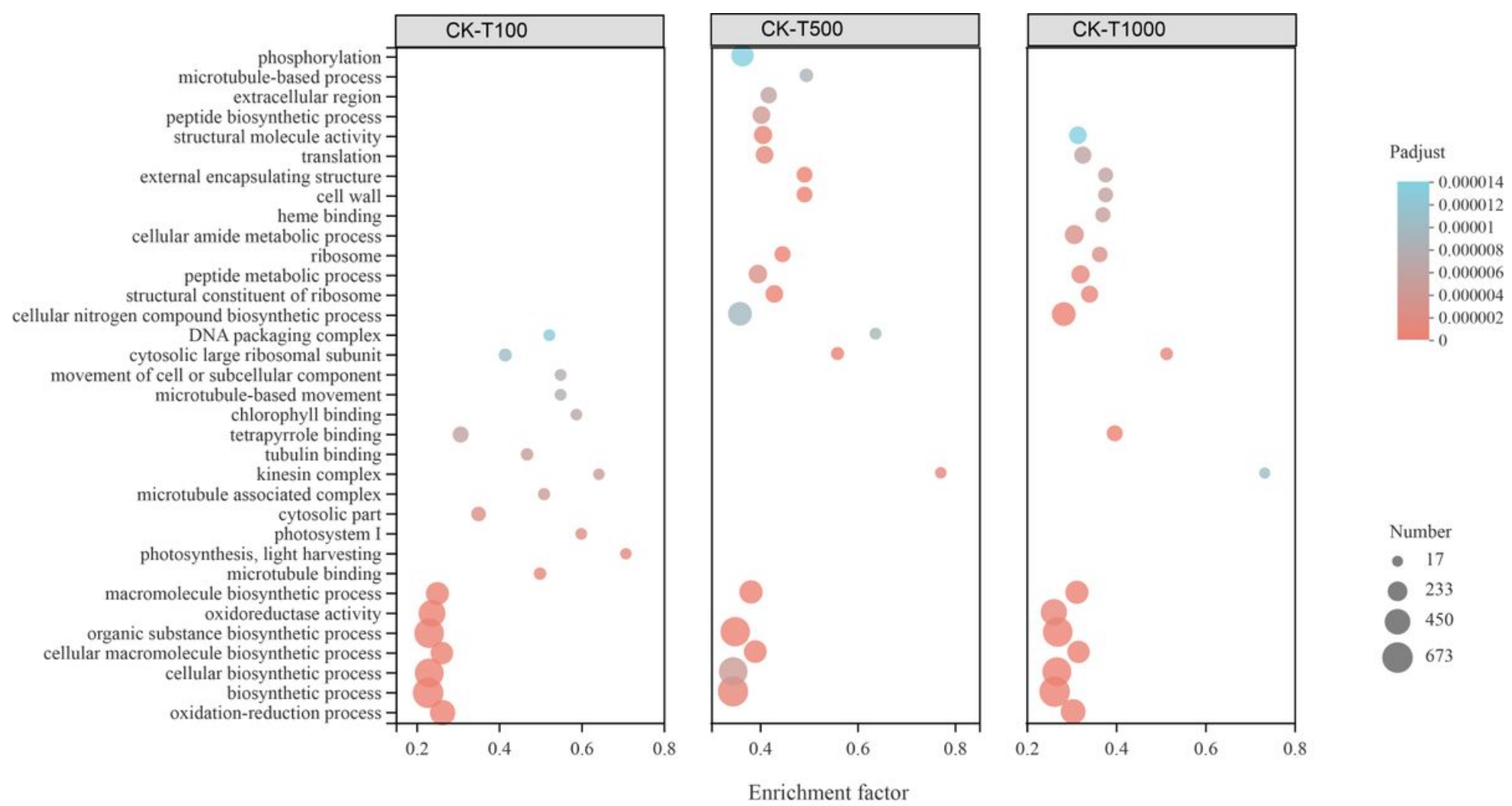

Figure 1

GO classification of unigene 


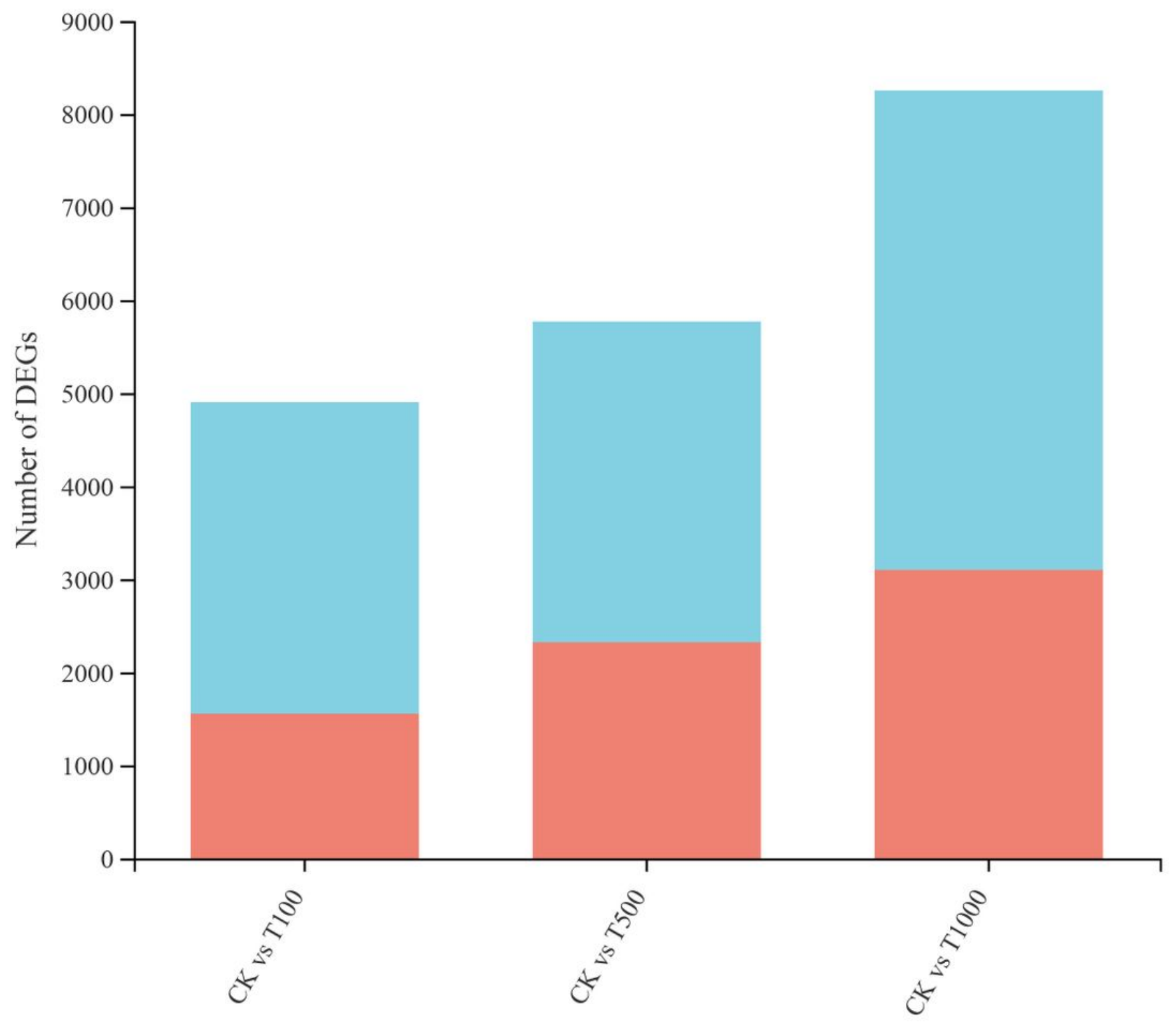

Figure 2

statistical results of expression difference among groups under antimony stress 
A

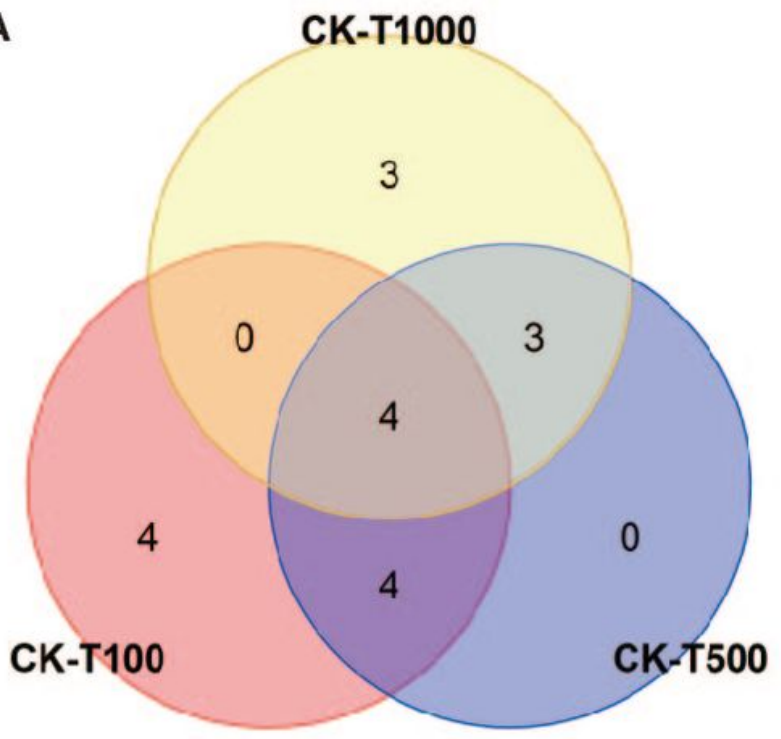

B

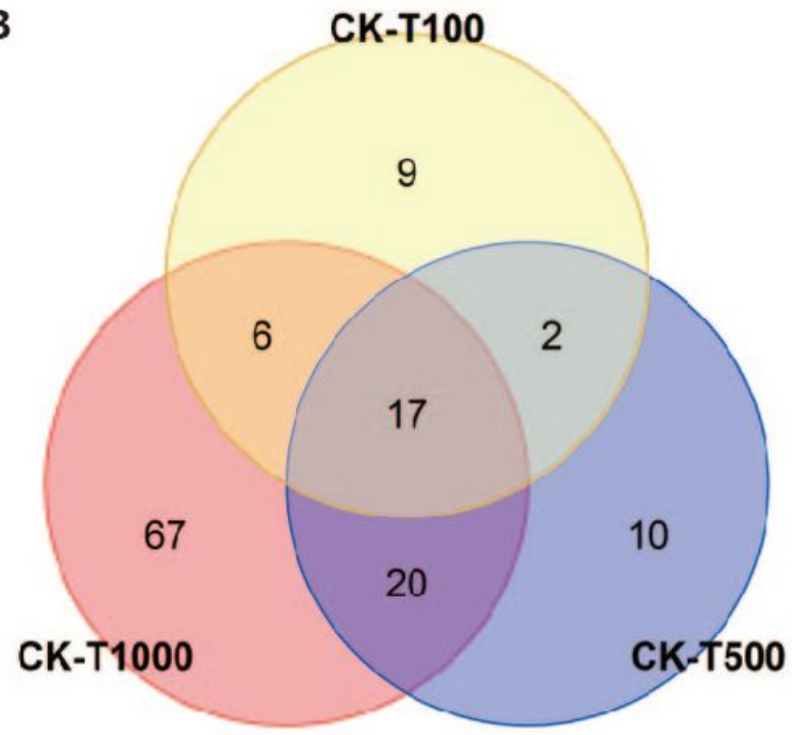

Figure 3

distribution of differential genes in each treatment group (| log2fc | 10) 


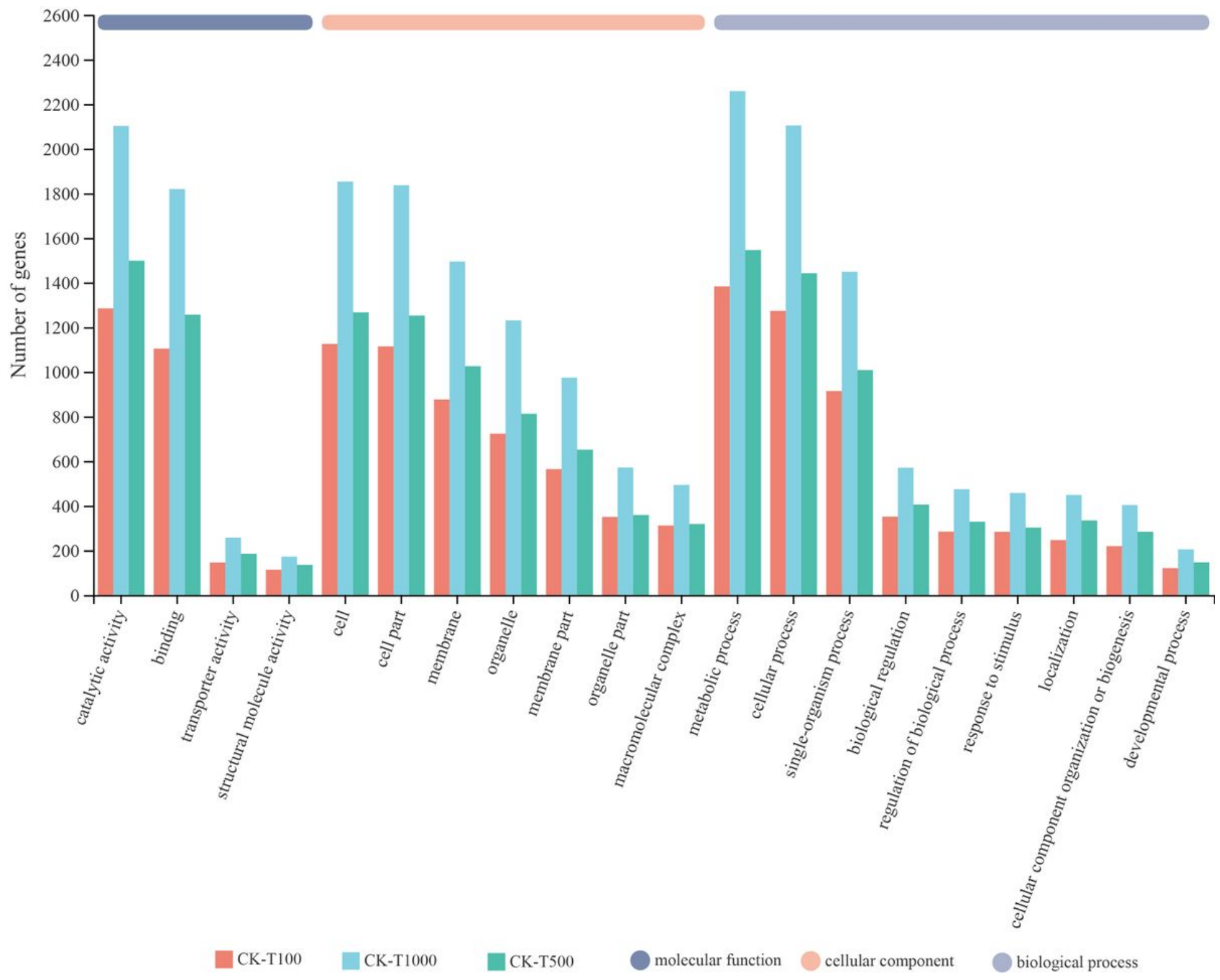

\section{Figure 4}

enrichment results of KEGG pathway under antimony stress Note: Y-axis: name of KEGG metabolic pathway; Xaxis: number of genes/transcripts annotated to this pathway. The seven categories of KEGG metabolic pathways include metabolism, genetic information processing, environmental information processing, cellular processes, organismal systems, human diseases, and drug development. (note: the number of categories differs among different species). 


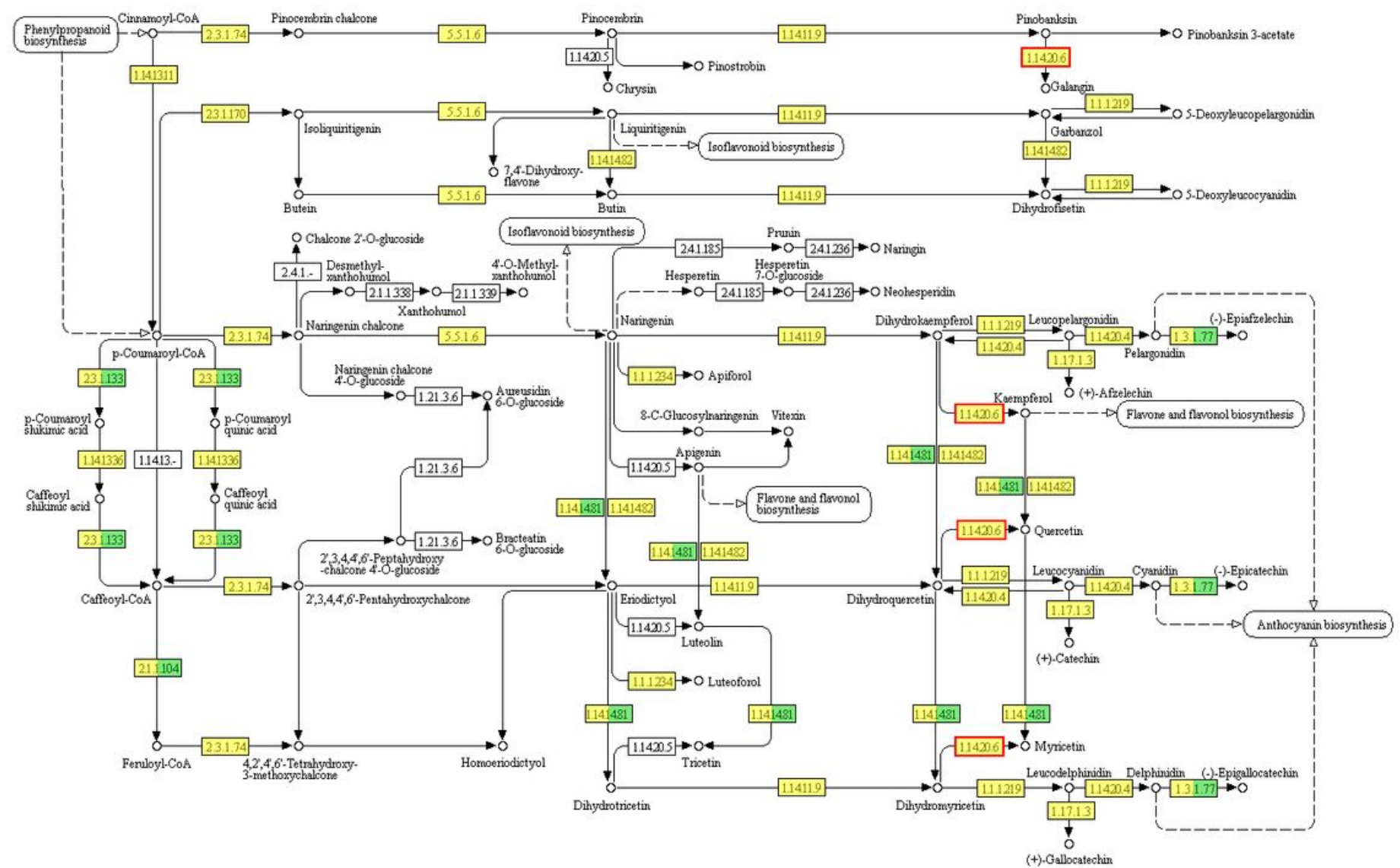

Figure 5

flavonoid synthesis pathway in response to antimony stress Note: yellow: the reference transcript; red frames: the upregulated transcripts; red yellow: reference gene transcripts and new gene transcripts. 